\title{
STUDIES ON ENERGY LOSS, RANGE AND STRAGGLING OF LIGHT IONS IN POLYMER (PMMA) FILM
}

\author{
Mahalesh Devendrappa ${ }^{1}$, Hajibaba K Inamdar ${ }^{2}$, Basavaraja Sannakki ${ }^{3}$ \\ ${ }^{1,2,3}$ Department of Post Graduate Studies and Research in Physics, Gulbarga University, Kalaburagi- 585 106, \\ Karnataka, India
}

\begin{abstract}
Energy loss of alpha particles of energy $5.48 \mathrm{MeV}$ [Am-241], has measured in Poly methyl methacrylate [PMMA] films at a pressure of 4.0-5.6 mbar. The experiment was carried out using the alpha ray spectrometer consisting of the Silicon Surface Barrier Detector. The alpha source and the detector were kept at a fixed distance of $16 \mathrm{~mm}$ in scattering chamber. The energy calibration of alpha particles in vacuum at a pressure of 4.6 mbar is $9.52 \mathrm{KeV} / \mathrm{channel}$. The value of energy loss found in the Poly methyl methacrylate film is $3.248 \mathrm{MeV}$ and the computed stopping power [SRIM 2008] for alpha particle in PMMA film is 1.798 $x 10^{2} \mathrm{MeV} / \mathrm{mm}$. The nuclear, electronic energy loss and projected range as a function of projectile energy in the range 2-10 MeV for alpha particles in PMMA is also studied using SRIM. It was found that the stopping power of alpha particles in PMMA film decreases exponentially as transmitted energy increases. Further the projected range of alpha particles increases exponentially as energy increases.
\end{abstract}

Keywords: Energy Loss, Poly Methyl Methacrylate Thin Film, Range, SSBD, Straggling And SRIM Code. $* * *$

\section{INTRODUCTION}

The energy loss of ions passing through matter has been the subject of exhaustive studies starting from the classical effort of Bohr, Bethe and Bloch and still remains motivating now. The study of the passage of fast-moving particles through matter has been important, since from the early days of nuclear physics. For light ions, such as ${ }^{4} \mathrm{He}^{+}$penetrating through a solid, the energetic particles lose their energy (Northcliffe and Schilling 1970, Hubert et al 1990 Ziegler 1999) primarily thorough the excitation and ionization in the inelastic collision with atomic electron termed as electronic energy loss. Microscopically, energy loss due to excitation and ionization is a discrete process. However, macroscopically, it is a good assumption that the moving ions lose their energy, continuously. We considered the average energy loss during the penetration of ions into a given material. The measured energy loss must be determined with the distance $\Delta \mathrm{t}$ or the thickness of the thin films, the ions will navigate through the target or thin films. The ions lose their energy, while passing through the target, which depends on the Mass Density ( $\rho$ ) and Atomic density $(\mathrm{N})$. The Mass Density and Atomic density together give rise to the Energy Loss of the target material i.e. $\rho \Delta \mathrm{t}$ or $\mathrm{N} \Delta \mathrm{t}$. Silicon Surface Barrier Detectors (SSBDs) have already been used in the fields of charged particle detection as a target for coherent interaction in a series of experiments by Bellini et al [1]. Yuan was probably first to use them for detection of charged particles. But, in general the solid state detectors have been used scanty for high energy physics experiments. Because, they cannot provide large detecting area, which is required in common Spectrometers. A need is required now for the thin particle detector very near to the interaction region with high precision high rate and high multiplicity capacity, which is difficult to meet with existing detectors. Especially, the study of very short lived charged particles will profit for an electronic detector in the target region [2]. The SSBDs are fabricated with N-type silicon with resistivity in the range $1000-8000 \mathrm{ohm}-\mathrm{cm}$, the silicon wafer after lapping to remove the surface damage created by crystal slicing process is cleaned with organic solvent[3]. The charge carrier lifetimes and nobilities are high, which is necessary for low noise detectors with a good timing behavior. Silicon surface barrier (SSB) radiation detectors are widely used in experimental nuclear physics for the spectroscopy of alpha particles, heavy ions, and fission fragments. The Schematic diagram of experimental arrangement is as shown in below figure 1 .

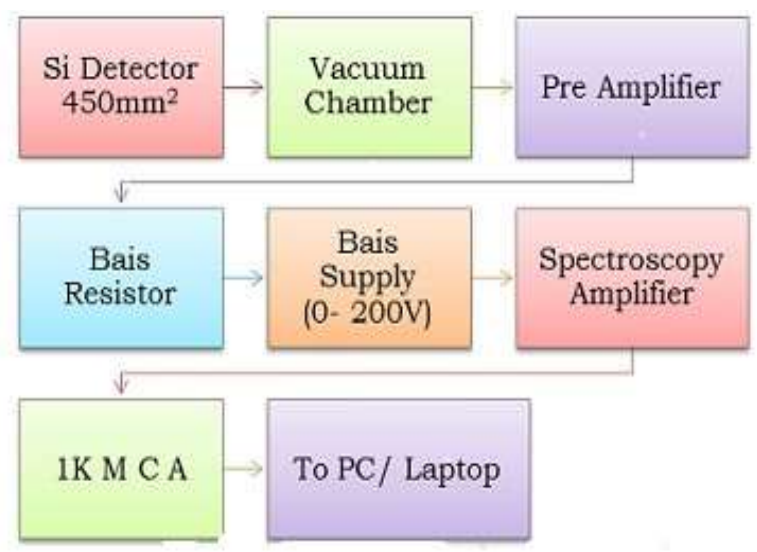

Fig -1 The block diagram experimental arrangement

\section{THEORETICAL BACKGROUND}

This theory was proposed by Bohr in 1948, it is based on the assumption that the incident ions are fully stripped and their energy loss in the target material is very small as compared 
to the incident ion energy [6]. According to this theory, the calculations of straggling standard deviations, Bohr developed the following expression.

$$
\left(\Omega_{B}^{2}\right)=4 \Pi\left(Z_{1}\right)^{2} e^{4} Z_{2} N x
$$

Where $Z_{1}$ and $Z_{2}$ are the atomic number of incident ion and target material respectively, $\mathrm{e}$ is the electronic charge, $\mathrm{N}$ is the number of target atoms per unit volume and $\mathrm{x}$ is the target thickness.

The alpha particles with initial energy $E_{1}$ enters into the medium [9] While, $E_{2}$ being the transmitted energy from the medium. The energy loss $[\Delta \mathrm{E}]$ of alpha particles can be obtained using equation. 2.

$$
\Delta E=E_{1}-E_{2}
$$

The stopping power per unit path length relation is given by fallowing equation. 3 .

$$
\Delta E / \Delta X \text { or } d e / d x
$$

\section{EXPERIMENTAL MEASUREMENT}

Preparation of thin film: Polymer substance of Poly methyl methacrylate was obtained from S.D. Fine Chem. Ltd, Mumbai, India which was in solid form. The films of Poly methyl methacrylate were prepared by solution casting method. In which the different prepositions of PMMA substances have been used to prepare the thin films at different thickness. The appropriate quantity of PMMA was dissolved in the acetone. The polymer solution was poured into a glass plate and kept for 48 hours at room temperature for evaporation of toluene and get thin layer of PMMA film on the glass substrate. The thin film was pealed out gently and used to measure the energy loss. The experiment has carried out using passivated ion-implanted planar SSBD of resolution $20 \mathrm{KeV}$ in vacuum for alpha particles [Am-241] of energy $5.48 \mathrm{MeV}$ connected with vacuum chamber using a hosepipe. The detector connected with vacuum chamber made with solid brass Nickel plating for ease of decontamination high performance O-ring seal. The PMMA thin film was mounted on the sample holder to measure the energy loss in the scattering chamber that has internal dimensions $61 \mathrm{~mm}$ wide $\times 74 \mathrm{~mm}$ deep $\times 10 \mathrm{~mm}$ high. The distance of the sample varies from $2 \mathrm{~mm}$ to $40 \mathrm{~mm}$ with in step of $4 \mathrm{~mm}$. The PMMA film was placed at a distance of $16 \mathrm{~mm}$ from the detector. The vacuum of 4.6 mbar pressure was created in the scattering chamber and it was controlled using three positions mentioned as PUMP, HOLD and VENT [8]. The operating voltage of +30 volts was applied to the SSBD detector through software of an alpha ray spectroscopy. The data acquisition was made using the computer interface ALSS software and stored in a computer. The energy calibration for the alpha particle at 4.6 mbar pressure is $9.52 \mathrm{KeV} / \mathrm{Channel}$.

\section{RESULT AND DISCUSSION}

The measured energy loss in Poly methyl methacrylate film at a pressure of 4 to $5.6 \mathrm{mbar}$ is $3.248 \mathrm{MeV}$ and in vacuum is $9.52 \mathrm{KeV}$ per channel and the computed stopping power is $1.798 \times 10^{2} \mathrm{MeV} / \mathrm{mm}$ this is due to the inelastic collisions with silicon electrons, which result in the charge pulse collected at the detector surfaces. The theoretical values have been confirmed at lower energies. The energy loss of alpha particles at different pressures of the vacuum medium is shown in figures [fig.]. As the pressure in the vacuum chamber increases, the alpha particle energy peaks widen. This is due to energy straggling, a process where statistical fluctuations occur in the number of collisions along the path of the particles and in the amount of energy lost per collision. It is observed from fig 2 . That as the pressure in scattering chamber increases the energy loss of the alpha particle decreases. This was ascribed to the lesser interaction of alpha particles with the medium. At higher pressure, the scattering chamber possesses less number of gaseous molecules in the traversing path of the alpha particle ions and hence the probability of the interaction will be less. As a result, this leads to a lesser energy loss of alpha particles in the medium. Consequently, the transmitted energy of alpha particles is high at higher pressure but whereas at lower pressure the transmitted energy is less [12-17]. This is due to the more number of molecules present in the scattering chamber and that result in the probability of interaction of the alpha particles with these molecules is higher and that gives the lesser transmitted energy. The fig 3. show a plot of nuclear and electronic energy loss in PMMA, as a function of projected energy of alpha particles in the energy range of 2-10 MeV, using SRIM [Verson-2008]. This graph reveals that as projected energy of the alpha particles increases the nuclear energy loss decreases exponentially. This is due to the interaction mechanism by which the ion can lose energy by elastic collision with the nuclei of target atoms of atmospheric air media.

The fig 4. show energy verses projected range and of alpha particles in atmospheric gas at room temperature from 2$10 \mathrm{MeV}$. It gives that as mean range of ions increases due to statistical fluctuations in the scattering chamber this leads increase in range of alpha particles in the air. This is in concurrence with the results of both Comfort et al. [18] and Mason et al. It is interesting to note that helium is the only gas for which the experimental results are much bigger than any one of the theoretical curves. The charge exchange phenomenon again offers a possible explanation. Ions with different charges will lose energy at different rates. Due to the capture and loss process an alpha particle will waste sometime in the singly charged state and sometime in the doubly charged state while passage the absorber. 
The time spent in one state or another will be different for different ions, and thus they will arrive at the detector with different energies; therefore, the net effect of the capture and loss process is to further broaden the distribution. It is reasonable to believe that charge exchange between alpha particles and the helium atoms will be relatively large since they have identical atomic excitation and ionization energies, eventually this leads to an anomalous large amount of straggling.

The Fig 5. shows the transmitted energy verses stopping power (Se) of an alpha particle at various pressures. It resulted that as transmitted energy of alpha particle increases the stopping power of alpha particles increases exponentially due to increase in the molecules of alpha particles in the traversing path; inside the scattering chamber this leads the comparison of electronic energy loss of gas molecules.

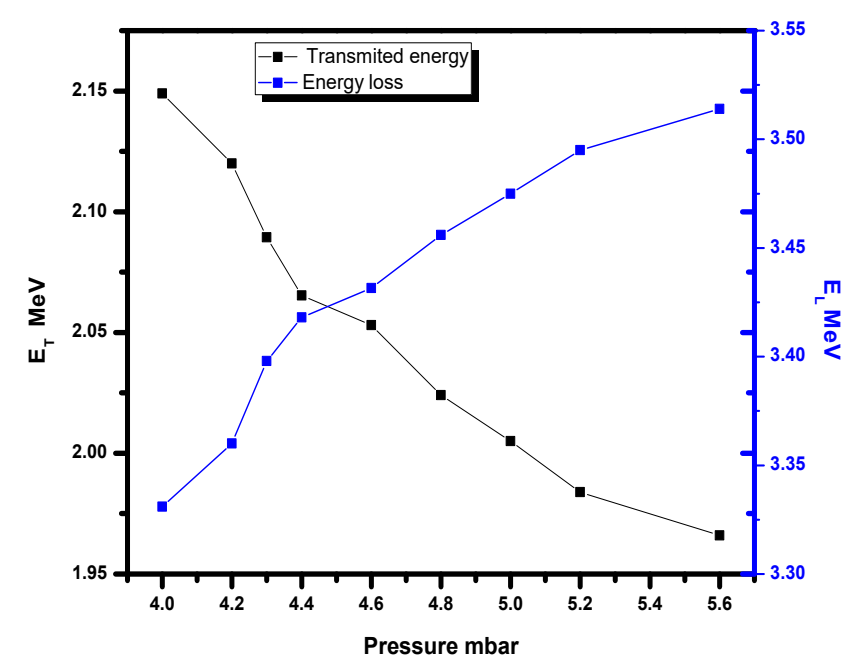

Fig -2 The plot of transmitted energy and energy loss versus pressure for light ions in PMMA film

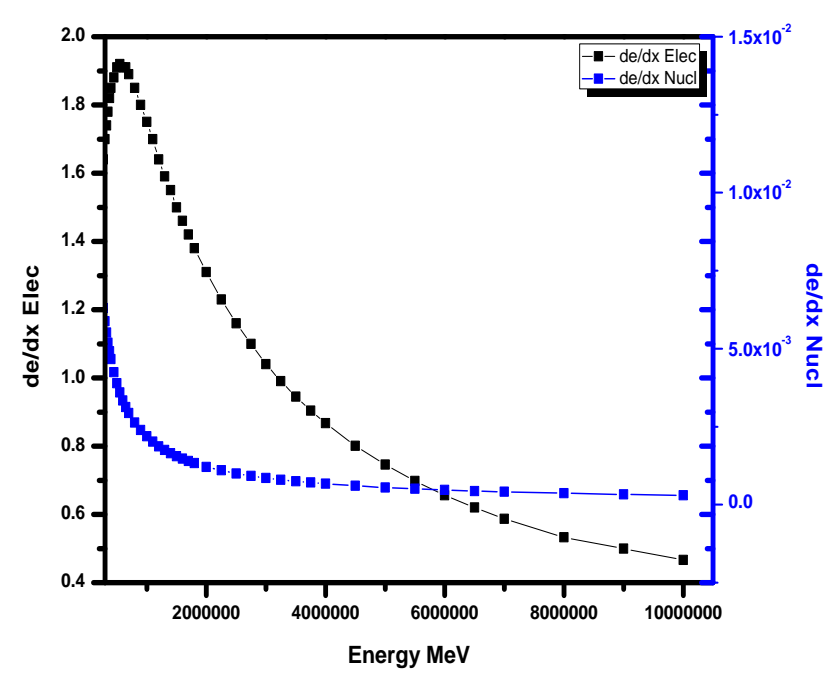

Fig -3 The plot of electronic energy loss and nuclear energy loss versus projectile energy of light ions in PMMA film

[Using SRIM 2008]

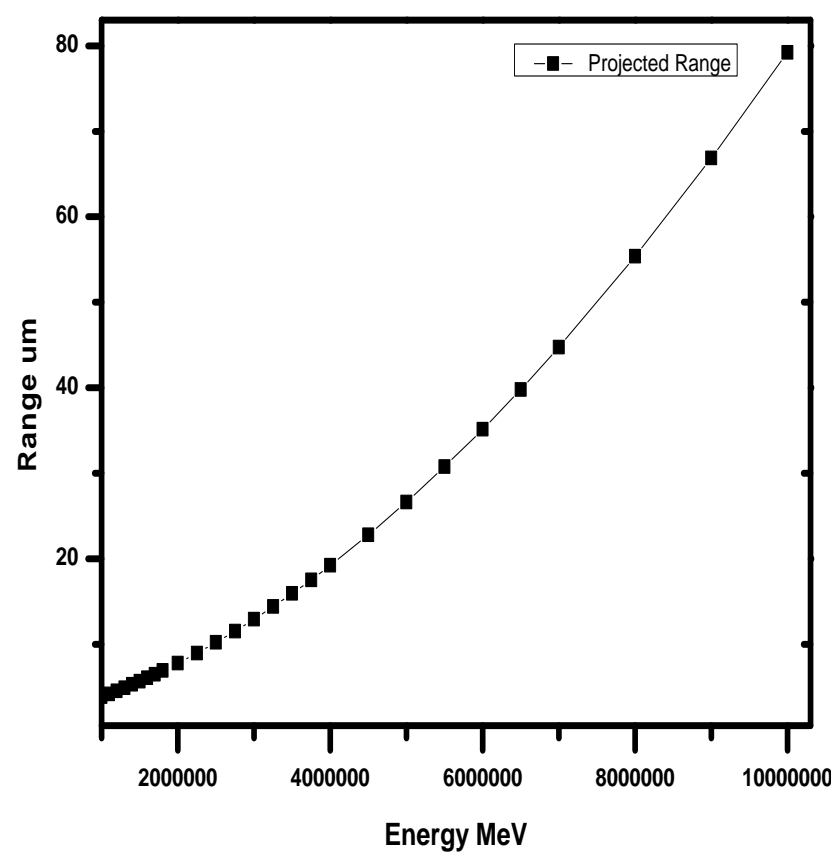

Fig -4 The plot of projected range versus energy of light ions in PMMA

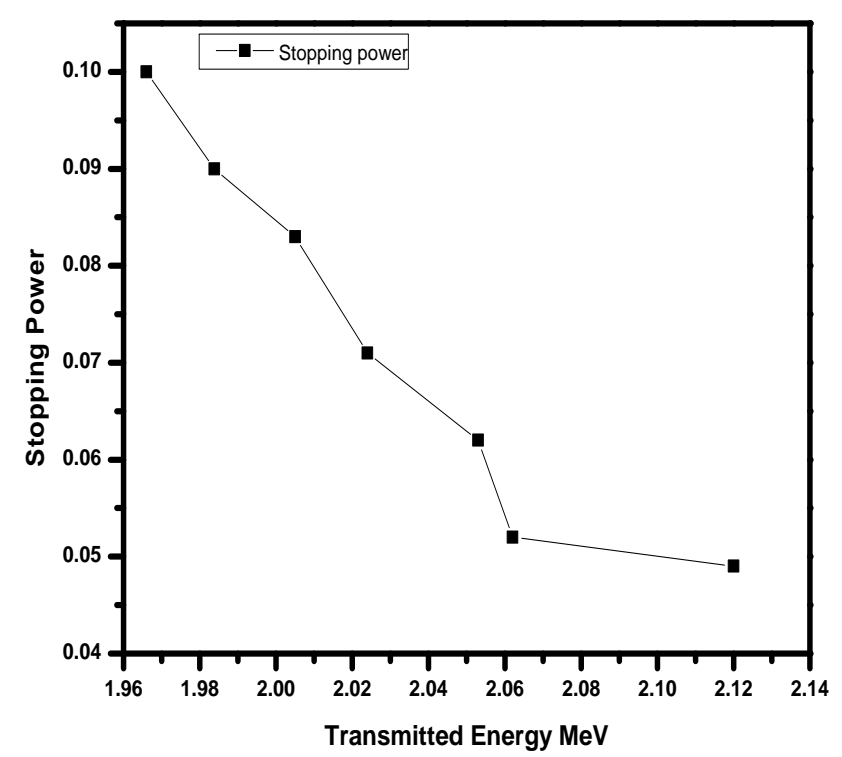

Fig -5 The plot of stopping power versus transmitted energy in PMMA

\section{CONCLUSION}

In conclusion, the measured energy loss of alpha particles decreases with an increase in the pressure of the scattering chamber. It was due to the reduction of air molecules in the scattering chamber or in the traversing path of the ions in the medium at higher pressures. This was resulting in a lesser energy loss at high pressures. Further, the transmitted energy of alpha particles increases with an increase in the pressure and that was resulted in the higher projected range of alpha particles. The Electronic, nuclear energy loss and range of alpha particles at atmospheric gas is also studied. In this case, as the transmitted energy of alpha particles increases the stopping power decreases exponentially. It can 
be observed that for low energies, the helium straggling results are much larger than the Bohr predictions, and with increasing ion energy; the helium experimental data approach the Bohr values. These results hold's good agreements with SRIM code computation.

\section{ACKNOWLEDGEMENT}

The above said authors are greatly indebted to Department of Physics, Gulbarga University, Kalaburagi and UGC New Delhi for providing a financial assistance to the MRP project.

\section{REFERENCES}

[1]. G Bellini et al., Nucl. Instr. and Meth. (1973), pp-107 .

[2]. J.F. Ziegler, J.M. Manoyan, Nucl. Instr. and Meth. B35 pp-215. (1988).

[3]. G. F. Knoll, Radiation Detection and Measurement (John Wiley and Sons, New York, 1999). pp- 354.

[4]. Alpha Spectroscopy Catalog, pp.2-4, (2011).

[5]. P.K. Diwan et al, Radiation Physics and Chemistry, vol81pp. 1543-1546, (2012).

[6]. N. Bohr, The penetration of atomic particles through matter. Kgl. Dan. Vid. Selsk. Mat. Fys. Medd. 18 (8), pp1144. (1948).

[7]. W.H.Bragg, and Kleeman, R.Philos. Mag. vol-10 S318. (1905).

[8]. Alpha spectroscopy user manual pp.1-3.9(2011).

[9]. E.H.M. Heyne et al., Nucl. Instr. and Meth.vol-178 pp.331-343, (1980).

[10]. J.Lindhard, M.Scharff, Energy loss in matter by fast particles of low charge. Kgl. Dan. Vid. Selsk. Mat. Fys. Medd. Vol-27 (15), pp.1-32. (1953).

[11]. N.Bohr,Mat. Fys. Medd. Dan. Vid. Selsk. 18, pp-8 (1948).

[12]. Basavaraja. Sannakki and Mahalesh Deveendrappa Int. Journal of Res in Enng \& Tech.vol-03, pp 286-289. (2014).

[13]. J. F. Ziegler Journal of Applied Physics/ Rev. App .Phys.Vol-85.pp1249-1272 (1999).

[14]. M.Zardo et $a$, Nucl. Instr. and Meth in Phys Resrch B. Vol-259, pp-836-840. (2007).

[15]. M. S. Livingston and H . A. Bethe, Rev . Mod. Phys . Vol-9, pp-245 (1937).

[16]. D. Jay Bourke and T. Christopher Chantele, Journal of Physical Chemestry A, vol-116, pp-3202-3205, (2012).

[17]. Mahalesh Devendrappa and Basavaraja Sannakki, Journal of Instrument Socity of India, Vol-44, pp-248-251, (2014).

[18]. J. R. Comfort, J. F. Decker, E. T. Lynk, M. O. Scully, and A. R. Quinton, Phys. Rev. 150, 249 (1966). 